\title{
A Case Study of Thiourea-Assisted Iminium Formation by Hydroxyl Anion Binding: Kinetic, Spectroscopic and Computational Evidences.
}

\author{
Veronica Juste-Navarro, ${ }^{\mathrm{a}}$ Liher Prieto, ${ }^{\mathrm{b}}$ Ignacio Delso, ${ }^{\mathrm{c}}$ Rubén Manzano, ${ }^{\mathrm{b}}$ Tomas \\ Tejero, ${ }^{\mathrm{a}}$ Efraim Reyes, ${ }^{\mathrm{b}}$ Jose L. Vicario, ${ }^{\mathrm{b} *}$ and Pedro Merino ${ }^{\mathrm{d} *}$
}

a Instituto de Síntesis Química y Catálisis Homogénea (ISQCH). Universidad de Zaragoza-CSIC. 5009 Zaragoza (Spain)

b Department of Organic Chemistry II. University of the Basque Country (UPV/EHU). P.O. Box 644, 48080 Bilbao (Spain). Phone: +34 94601 5454. E-mail: joseluis.vicario@ehu.es

c Servicio de RMN. Centro de Química y Materiales de Aragón. Universidad de Zaragoza-CSIC. 5009 Zaragoza (Spain)

d Instituto de Biocomputación y Física de Sistemas Complejos (BIFI). Universidad de Zaragoza-CSIC. 5009 Zaragoza (Spain)- Phone: +34 87655 3783. E-mail: pmerino@unizar.es

Received: ((will be filled in by the editorial staff))

Supporting information for this article is available on the WWW under http://dx.doi.org/10.1002/adsc.201\#\#\#\#\#\#.

\begin{abstract}
The experimental and computational study of the mechanism of the iminium-organocatalyzed formation of $\mathrm{N}$ hydroxypyrrolidines from nitrones, as a case study, revealed a hitherto unknown mode of action for the Schreiner's thiourea used as co-catalyst. The experimental data suggest that thiourea promotes the formation of the iminium ion through hydroxyl anion recognition forming a stable ion pair. Such an ion pair facilitates enolization of the nitrone through a H-bond network thus promoting the reaction with iminium ion. The computational model supports the mechanism and the catalytic cycle. This mechanistic rationale is supported by the lack of reactivity of preformed iminium ion with the nitrone in the absence of thioureahydroxyl complex and the observed reactivity when a complex thiourea-tetrabutylammonium hydroxide is added.
\end{abstract}

Keywords: Thioureas; Iminium catalysis; Nitrones; Hydroxide binding; Squaramides

Iminium catalysis has emerged over the past twenty years as one of the most powerful methods in organocatalysis. ${ }^{[1]}$ When a carbonyl compound is placed in the presence of a secondary amine, an equilibrium with the hemiaminal HA is established. HA can lead to the corresponding iminium ion IM but this process is not spontaneous (Scheme 1). ${ }^{[2]}$.

In particular cases, a reagent can assist -by means of $\mathrm{H}$-bond interactions- the elimination of the hydroxyl group and subsequent iminium formation, as in the case of an oxa-Michael reaction recently reported from our laboratories. ${ }^{[3]}$ Depending on substrates and reaction, the formation of the iminium ion might not be strictly required, ${ }^{[4]}$ although it is a rare circumstance. In any case, the commonest situation is that a Brønsted acid (AH) helps to form IM by protonation of the hydroxyl group of the hemiaminal. In fact, iminium catalysis generally involves a secondary amine and an acid co-catalyst, the iminium ion forming an ion pair with the counteranion of the acid. By using strong acids such as trifluoroacetic acid or perchloric acid, iminium ions have been isolated and characterized. ${ }^{[5]}$ In this respect, the non-nucleophilic character of the counterion is crucial. ${ }^{[6]}$

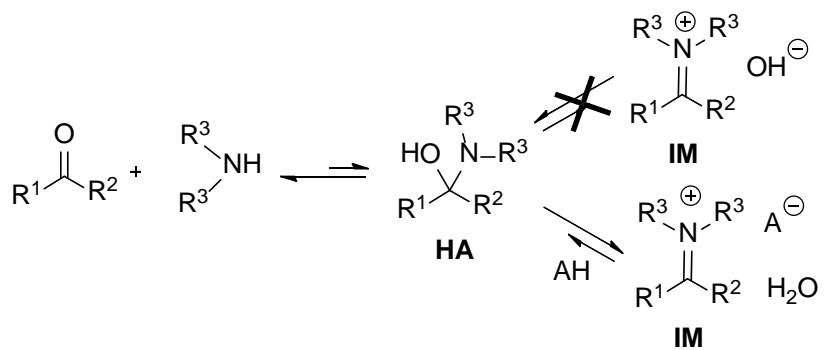

Scheme 1. Formation of iminium ions assisted by a Brönsted acid.

The reversibility of the process illustrated in Scheme 1 is often a drawback for many organocatalytic reactions requiring long reaction times due to the limited availability of the iminium ion. In order to increase the concentration of iminium ion IM, the use of thioureas capable of sequestering the counteranion (typically a benzoate) has been reported by $\mathrm{Xu}$ and co-workers. ${ }^{[7]}$ Indeed, thioureas are known as reagents for anion recognition. ${ }^{[8]}$ Organocatalytic activity of thioureas, ${ }^{[9]}$ including their role in bifunctional catalysts, ${ }^{[10]}$ is closely related with their role in molecular recognition processes. ${ }^{[11]}$ Jacobsen and co-workers reported that recognition of chloride by an amidothiourea was crucial for generating the required iminium ion in an asymmetric Pictet-Spengler-type reaction (Scheme 
2). ${ }^{[12]}$ In general, thioureas act as co-catalysts either facilitating the catalytic action of the chiral catalyst or activating one of the reagents, ${ }^{[13]}$ typically electrophiles such as carbonyl compounds, imines and nitroalkenes, among others, against nucleophiles. ${ }^{[14]}$

$$
\text { (Jacobsen, 2009) }
$$

Scheme 2. Recognition of anions by thiourea.

In this work, we report experimental and computational evidences of hydroxyl anion recognition by Schreiner's thiourea 3 promoting the formation of iminium salt $\mathbf{4}$ from amine $\mathbf{1}$ and aldehyde $\mathbf{2}$ without any additional acid co-catalyst (Scheme 2). The formation of counterion 6 agrees with previous investigations reporting anion-induced partial deprotonation of (thio)ureas. ${ }^{[15]}$ and with the only reported recognition of hydroxyl anion by a thiourea. ${ }^{[16]}$ We chose as a case study our recently reported reaction of $\mathbf{2}$ with nitrones 7, catalyzed by $\mathbf{1}$ leading to $N$-hydroxypyrrolidines 8 (Scheme 2) ${ }^{[17]}$ The crucial role of ionic pair $\mathbf{4 \cdot 6}$ for the reaction and the full mechanism, including the catalytic cycle, have also been studied experimental- and computationally. The effect of hydrogen bonds, and the factors that influenced the stereochemistry were explored to get useful information on organocatalytic reactions of nitrone ylides and the potential role of thiourea in iminium catalysis.

Both in our preliminary communication ${ }^{[17]}$ and a previous similar study with isatin-derived nitrones, ${ }^{[18]}$ triethylamine was used to deprotonate the nitrone $\mathbf{7}$ and generate the nitrone ylide assuming a mechanism involving the anion derived from 7. Under this hypothesis the role of thiourea 3 was limited to activate the carbonyl group in the first step and/or the nitrone moiety in the second one. ${ }^{[19]}$ However, a more in-depth analysis of the reaction conditions showed that the reaction with nitrones 7 does not require the presence of any base. On the other hand, the presence of thiourea $\mathbf{3}$ in catalytic amounts was essential for the progress of the reaction, which does not work in the absence of 3. The results in the absence of triethylamine were similar in yield and identical in $\mathrm{dr}$ and ee to those obtained in the presence of triethylamine (see SI). ${ }^{[17]}$

To investigate in detail the mechanism of the reaction we carried out some kinetic analysis of the reaction (see SI). We used as representative substrates nitrone 7a and aldehyde 2a (Scheme 3) and determined that the reaction is first-order in both $\mathbf{7 a}$ and 2a. The kinetic measurements also confirmed the reaction to be first-order in $\mathbf{1}$ and $\mathbf{3}$. The observed linear effects between ee and catalyst loading confirmed that the active catalyst is a monomeric species.

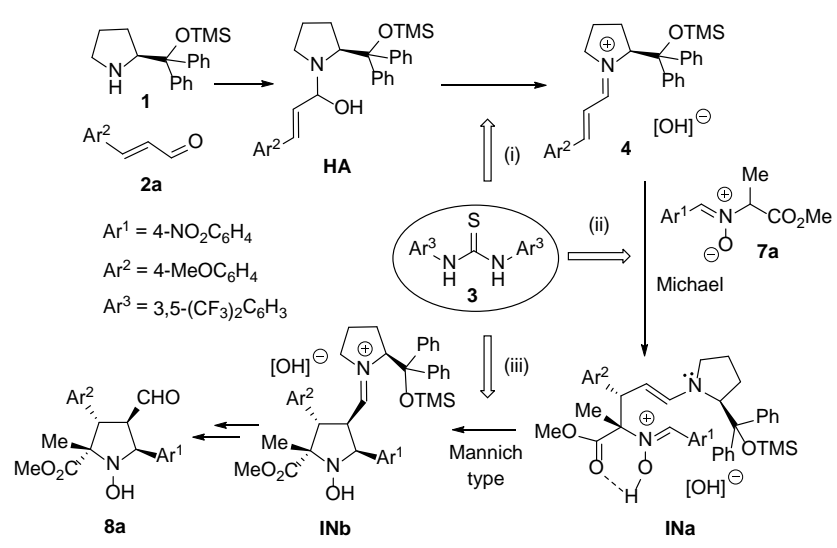

Scheme 3. Reaction between 2a and 7a in the presence of $\mathbf{1}$ and possible steps in which thiourea $\mathbf{3}$ might be involved. (i) formation of the iminium ion; (ii) enolization of nitrone and (iii) activation of nitrone in Mannich step.

The first-order dependence on both aldehyde and nitrone observed experimentally indicates that both reactants are not associated in the resting state, suggesting a typical mechanism via formation of an iminium ion. Once the iminium ion is formed, the reaction can take place in a concerted way or in two steps through a tandem Michael-Mannich reaction as we previously reported for electron-poor alkenes. ${ }^{[20]}$ In principle, thiourea activation can occur at different non-exclusive levels, i.e.: (i) by promoting the formation of the iminium ion; (ii) by facilitating enolization of the nitrone required for both mechanisms, considering that the reaction does not need the presence of a base, and (iii) by activating the nitrone towards the electrophilic addition. The stepwise mechanism, involving an initial nucleophilic attack to the iminium ion followed by a second nucleophilic attack of the intermediate enamine to the nitrone moiety, should be much more sensitive to electronic effects at the substituents. To evaluate such effects, we measured the rates of the reaction for several substituted nitrones and aldehydes. Hammett plots revealed a clear dependence of the electronic effects of the substituents indicating that the most favorable situation corresponds to nitrones with electron-withdrawing groups and aldehydes with electron-donating groups (ee SI). These data provide 
compelling evidence in favor of a stepwise mechanism based on nucleophilic attacks to iminium intermediate and nitrone (Scheme 3). ${ }^{[21]}$

The whole process, in which the precise involvement(s) of thiourea should still be investigated, can be divided in three parts, i.e: 1) formation of the iminium ion from the precursor hemiaminal HA, 2) reaction between iminium ion and nitrone and 3) formation of the final product.

Firstly, we studied the formation of the iminium ion. Combination of catalyst $\mathbf{1}$ with aldehyde $\mathbf{2 a}$ in 1:1 ratio lead to a mixture in which the signal ratios of both reagents remained unchanged without detection of any new signal by ${ }^{1} \mathrm{H}$ NMR spectroscopy (Figure 1, A). On the other hand, addition of 1 equiv of thiourea 3 resulted in the formation of iminium ion $\mathbf{4}$ as evidenced by comparing the observed signals in ${ }^{1} \mathrm{H}$ NMR (Figure 1, B1-B4) with those observed for the same iminium prepared as reported (Figure $1, \mathrm{C}){ }^{[5]}$

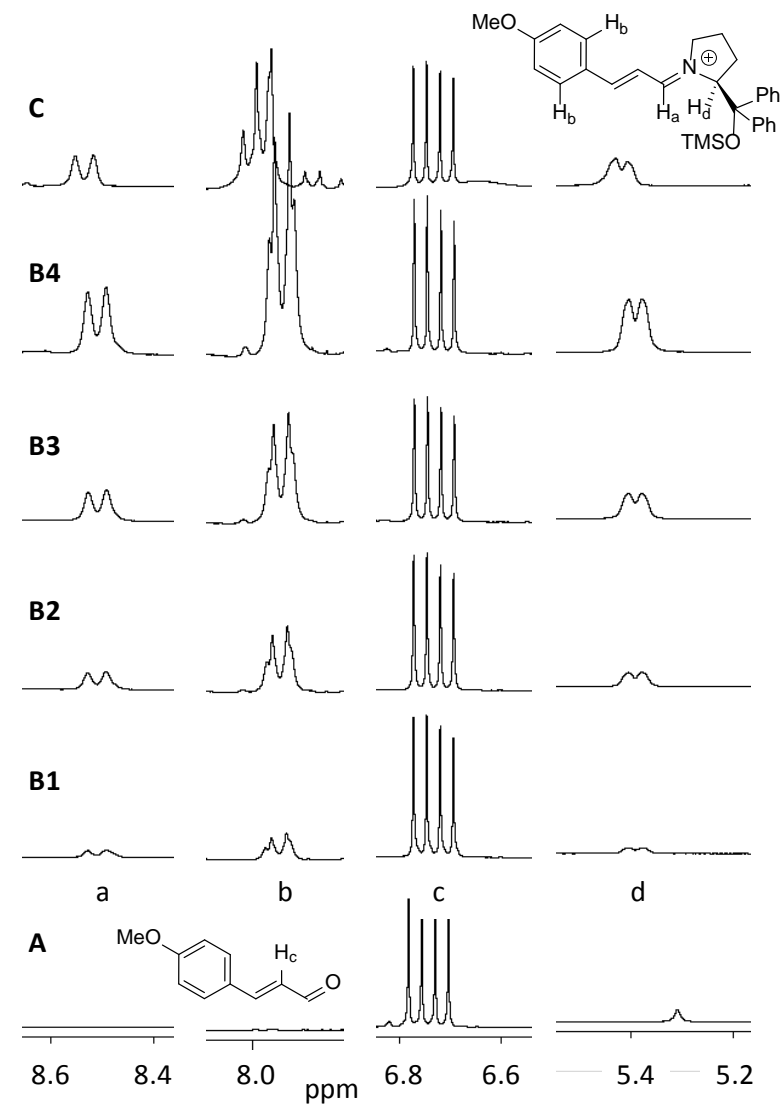

Figure 1. Selected regions of ${ }^{1} \mathrm{H}-\mathrm{NMR}$ spectra (for full spectra see SI). A: Equimolar mixture of 1 and 2a. B1-B4: A after adding 1.0 equiv of thiourea 3; B1: after $1 \mathrm{~h}$; B2: after 2 h; B3: after 6 h; B4: after 10 h. C: Iminium ion prepared from 1, 2a and trifluoroacetic acid as reported. ${ }^{[5]}$

Moreover, when the reaction mixture was submitted to ESI-MS strong signals at $\mathrm{m} / \mathrm{z} 470.2584$ and $\mathrm{m} / \mathrm{z}$ 499.0214 were observed corresponding to the iminium cation $(\mathrm{m} / \mathrm{z}$ calcd 470.2510$)$ and the thiourea anion $(\mathrm{m} / \mathrm{z}$ calcd 499.0144$)$ resulting from 6 after loss of water. The binding properties of thiourea 3 towards hydroxide anion have been evaluated and a value of $\log K=5.5 \pm 0.20$ was obtained (see SI) in agreement with a previous report for other thioureas. ${ }^{[16]}$

The binding of thiourea $\mathbf{3}$ to hydroxide ion was corroborated by monitoring the changes in the ${ }^{1} \mathrm{H}$ NMR spectrum of 3 upon addition of $\mathrm{Bu}_{4} \mathrm{NOH}$ solutions. Complete disappearance of both $\mathrm{NH}$ signals was observed after the addition of 1.0 eq of $\mathrm{Bu}_{4} \mathrm{NOH}$ (see SI). As expected, addition of nitrone $7 \mathbf{a}$ to the reaction mixture in which the ion pair $\mathbf{4 \cdot 6}$ had been identified resulted in the rapid formation of the $\mathrm{N}$ hydroxypyrrolidine 8a. From these results, it became evident that thiourea 3 is capable of promoting the formation of the iminium ion without the help of any acid co-catalyst.

Then we studied the second part of the catalytic cycle, i.e. the reaction of iminium ion with the nitrone. Remarkably, when nitrone 7a was added to a freshly prepared ${ }^{[5]}$ solution of iminium perchlorate $\mathbf{4} \cdot \mathbf{C l O}_{4}$ no reaction was observed after 2 days. ${ }^{[22]}$ The situation was the same when up to 1.0 equiv of thiourea was added. The addition of a base $\left(\mathrm{Et}_{3} \mathrm{~N}\right)$ resulted in a very slow reaction (20\% of conversion after 4 days). On the contrary, when a solution of a 1:1 mixture of thiourea 3 and $\mathrm{Bu}_{4} \mathrm{NOH}$ was added, the reaction proceeded as in the conditions reported in SI. These results suggest a second point of activation of the reaction, indicating that the formation of ion pair $\mathbf{4 \cdot 6}$ is required presumably for promoting enolization of nitrone.

Finally, we studied the formation of the final product. The process is more efficient with the ion pair 4.6 than using a base for generating the enolate of $\mathbf{7 a},{ }^{[23]}$ but an enolate should be more nucleophilic and should favor the second step of the reaction (Mannichtype). However, Mannich-type reactions with nitrones require activation of the nitrone; ${ }^{[24]}$ the higher efficiency of ion pair $\mathbf{4 \cdot 6}$ suggests a third point of activation of the reaction, i.e. the presence of a Hbonding network responsible for activating the nitrone towards the intramolecular Mannich-type reaction. We also monitored the reaction with ESI-MS and after 1 day of reaction signals at $\mathrm{m} / \mathrm{z} 470.2672$ and $\mathrm{m} / \mathrm{z}$ 722.3203 were observed corresponding to iminium cations $4(\mathrm{~m} / \mathrm{z}$ calcd 470.2510$)$ and that integrating INb ( $\mathrm{m} / \mathrm{z}$ calcd 722.3256$)$, definitively confirming the process illustrated in Scheme 3.

With the aim of giving support to our hypotheses and to propose precise structures of the species involved in the catalytic cycle considering thiourea participation, we carried out a computational study of the whole catalytic process at B3LYP-3DBJ/def2SVP level of theory considering solvent effects (see SI for details). We used for our model pyrrolidine as the catalyst, and phenyl rings at both nitrone and aldehyde. ${ }^{[25]}$. The proposed catalytic cycle according to calculations is given in Scheme 4. The cycle begins with the formation of complex HE from the catalyst $\mathbf{P Y}$, aldehyde AL and thiourea $\mathbf{3}$. The formation of iminium ion pair INO is favored by $10.8 \mathrm{kcal} / \mathrm{mol}$ in agreement with the experimental observations. The higher stability of INO is also due to the presence of favorable $\pi, \pi$-interactions between the pheyl ring of 
the iminium ion and one of the aromatic rings of thiourea 3 (see SI).

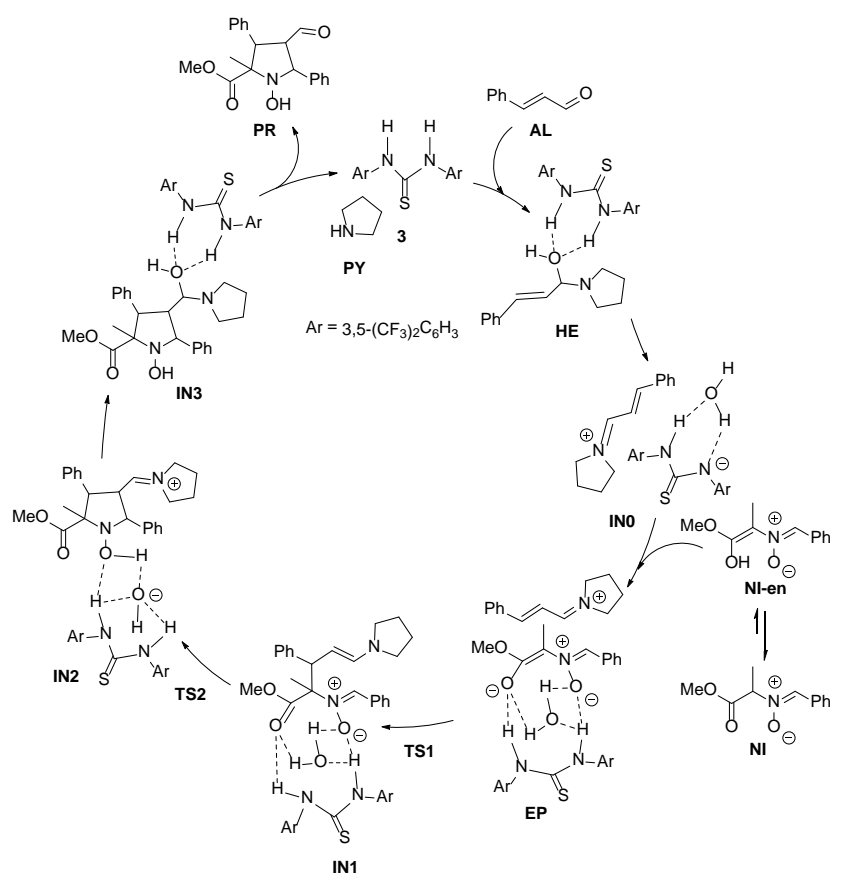

Scheme 4. Catalytic cycle for the reaction between AL and NI in the presence of $\mathbf{P Y}$ and $\mathbf{3}$.

Incorporation of nitrone NI results in the formation of encounter pair EP in which de counteranion of the iminium salt stabilizes the enol tautomer of the nitrone. After the first transition state, TS1, located at 3.1 $\mathrm{kcal} / \mathrm{mol}$ and identified as the rate-limiting step, the enamine intermediate IN1 is formed. This intermediate is ready for the next step through a second transition state, TS2, located at $1.8 \mathrm{kcal} / \mathrm{mol}$. In this transition structure thiourea facilitates a H-bond network responsible of activating the nitrone moiety towards the intramolecular nucleophilic attack. This step yields IN2 formed by a new iminium ion (detected by ESI-MS) having complex $\mathbf{6}$ as counteranion which interacts with the hydroxyamino functionality through H-bonds. Intermediate IN2 evolves to hemiaminal IN3, a transformation thermodynamically favored by $4.4 \mathrm{kcal} / \mathrm{mol}$ (for the preferred diastereomer). Releasing of product $\mathbf{P R}$ regenerates catalyst $\mathbf{P Y}$ and co-catalyst 3 . The driving force of the catalytic cycle is determined by the regeneration of HE from IN3 with concomitant release of the final product. This final catalyst-turnover step involves a favored energy of $\Delta \mathrm{G}=-22.8 \mathrm{kcal} / \mathrm{mol}$ (for a complete energy profile see SI). Two approaches of the iminium ion to the activated enolized nitrone are possible in EP, leading to two diastereomers. The corresponding transition structures TS1a and TS1b differ in $2.4 \mathrm{kcal} / \mathrm{mol}$ in favor of that leading to $\left(2 S^{*}, 3 S^{*}, 4 R^{*}, 5 S^{*}\right)$-isomer, in excellent agreement with the experiments. The almost complete enantioselectivity is due to the essentially exclusive attack by the less hindered face of the iminium formed from catalyst $\mathbf{1}$ as reported elsewhere. ${ }^{[25]}$

The calculated catalytic cycle is in good agreement with the experimental observations, including ESI-MS monitoring that allowed identifying iminium ions from IN0 and IN2. Calculations also support the three keypoints of the reaction in which thiourea acts on the reaction, i.e: promoting formation of iminium ion, nitrone enol and activating nitrone functionality in the second step.

In addition to $\mathbf{3}$ we also studied the formation of iminium 4 mediated by thioureas 9 and 10, and squaramides $\mathbf{1 1}$ and $\mathbf{1 2}$. The observed rates for $\mathbf{3}$ and 10-12 (no reaction was observed with 9) are illustrated in Figure 2. Although the formation of iminium ion was not complete, the best result (ca. 56\%) was found for the most acidic squaramide 12. Indeed, the iminium formation showed to be dependent on the $\mathrm{p} K_{\mathrm{a}}$ (we determined $\mathrm{p} K_{\mathrm{a}}$ for $\mathbf{1 2}$, see $\mathrm{SI}$ ), the higher acidity, the higher rate of iminium formation. The amount of iminium at the equilibrium was found to be linearly dependent on the relative stability between the iminium and the precursor hemiaminal. ${ }^{[26]}$

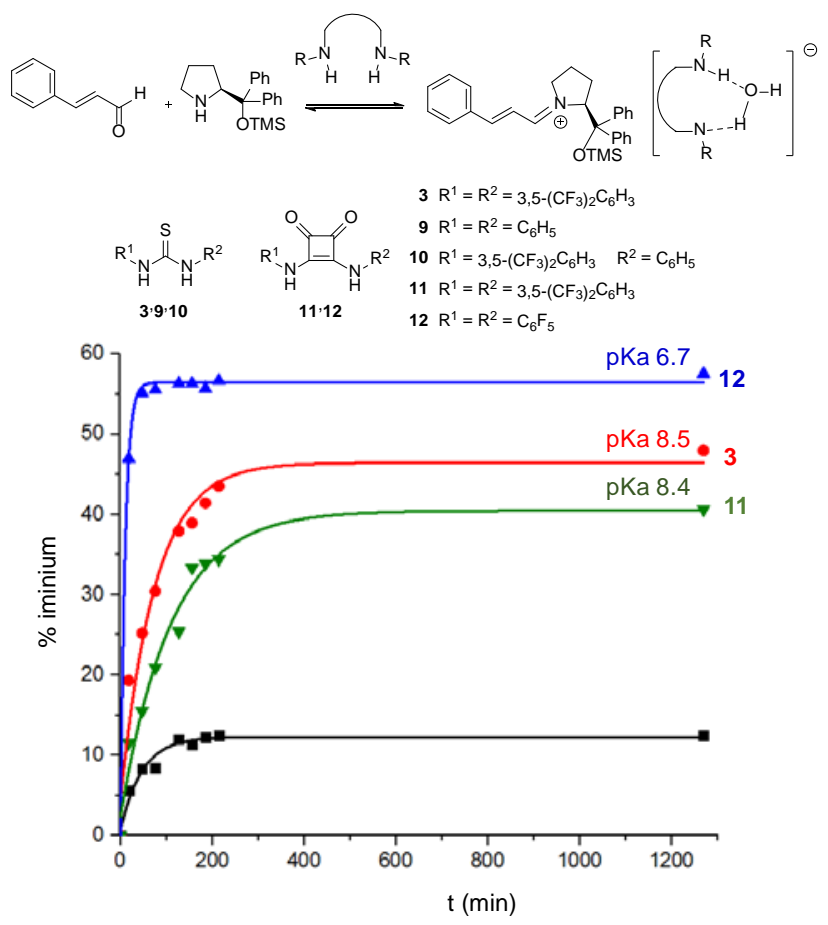

Figure 2. Reaction rates for the formation of iminium ion 4 from catalyst $\mathbf{1}$ and cinnamaldehyde $\mathbf{2 a}$ in the presence of thioureas 3, 9 and 10, and squaramides $\mathbf{1 1}$ and $\mathbf{1 2}$.

The reaction between 2a and 7a catalyzed by $\mathbf{1}$ in the presence of squaramides $\mathbf{1 1}$ and $\mathbf{1 2}$ led to essentially identical results in yield and enantioselectivity without observable effects in the rate of the reaction. This result agrees with calculations, which showed TS1 (and not the formation of the iminium ion) as the rate-limiting step. Accordingly, 
the observed rates of iminium ion formation for thiourea $\mathbf{3}$ and squaramides $\mathbf{1 1}$ and $\mathbf{1 2}$ are enough for feeding the catalytic cycle.

In summary, we have demonstrated that thioureas and squaramides can promote the formation of iminium ions without the presence of a Brønsted acid. The case study has demonstrated that hydroxide anion recognition is the main driving force helped by $\pi, \pi$ interactions between aromatic rings of co-catalyst and iminium, which thermodynamically favors the formation of the ionic pair INO (formed by iminium ion 4 and complex $[3 \cdot \mathrm{OH}]^{-}$) from the precursor hemiaminal (HE) in equilibrium with the reagents. Because of the absence of those hydrophobic interactions in the final iminium ion (IN2), the formation of hemiaminal (IN3) is thermodynamically favoured closing the catalytic cycle. The formed anion $[3 \cdot \mathrm{OH}]^{-}$has an additional effect facilitating enolization of the nitrone and promoting the attack to the iminium ion 4; this step is the rate-limiting step (TS1).

Consequently, it is possible to predict that the use of thioureas or squaramides might promote cascade reactions starting and ending with iminium ions, which should be formed and hydrolyzed, respectively. The formation of anion [3. OH] , which can be considered a weak base with capability of promoting H-bond networks, could also exert additional effects on the substrates. In this respect, it should of great interest to consider reagents requiring enolization or any kind of H-bond activation.

It is expected that these findings, in particular generation of iminium ions in a neutral or weakly basic medium, will allow evaluating the significance of iminium formation in a variety of organocatalytic reactions. Moreover, the observation of the same behaviour with squaramides expands considerably the number of organocatalytic processes susceptible to be modulated.

\section{Acknowledgements}

This research was supported by the Spanish MINECO (FEDERCTQ2016-76155-R, to PM and FEDER-CTQ2014-52107-P, to J.L.V.), the Government of Aragón (Grupos Consolidados, E.10) and the Basque Government (Grupos IT328-10), UPV/EHU (UFI QOSYC 11/22). V.J. thanks MEC for a pre-doctoral FPI fellowship. The authors acknowledge the resources from the supercomputers "Memento" and "Cierzo", technical expertise and assistance provided by BIFI-ZCAM (Universidad de Zaragoza, Spain).

\section{References}

[1] (a) A. Erkkilä, I. Majander, P. M. Pihko, Chem. Rev. 2007, 107, 5416-5470. (b) D. W. C. MacMillan, A. J. B. Watson, in Science of Synthesis, Asymmetric Organocatalysis, Vol. 1, Georg Thieme Verlag, 2012, pp. 309-401.

[2] Y.-Y. Huang, C. Cai, X. Yang, Z.-C. Lv, U. Schneider, ACS Catalysis 2016, 6, 5747-5763.
[3] A. Orue, U. Uria, D. Roca-López, I. Delso, E. Reyes, L. Carrillo, P. Merino, J. L. Vicario, Chem. Sci. 2017, 8, 2904-2913.

[4] A. Capobianco, A. Russo, A. Lattanzi, A. Peluso, Adv. Synth. Catal. 2012, 354, 2789-2796.

[5] H. Gotoh, T. Uchimaru, Y. Hayashi, Chem. Eur. J. 2015, 21, 12337-12346.

[6] (a) D. Seebach, R. Gilmour, U. Groselj, G. Deniau, C. Sparr, M.-O. Ebert, A. K. Beck, L. B. McCusker, D. Sisak, T. Uchimaru, Helv. Chim. Acta 2010, 93, 603634. (b) U. Groselj, D. Seebach, D. M. Badine, W. B. Schweizer, A. K. Beck, I. Krossing, P. Klose, Y. Hayashi, T. Uchimaru, Helv. Chim. Acta 2009, 92, 1225-1259.

[7] Y. Wang, T. Y. Yu, H. B. Zhang, Y. C. Luo, P. F. Xu, Angew. Chem. Int. Ed. 2012, 51, 12339-12342.

[8] D. E. Gomez, L. Fabbrizzi, M. Licchelli, E. Monzani, Org. Biomol. Chem. 2005, 3, 1495-1500.

[9] (a) M. Kotke, P. R. Schreiner, in Hydrogen Bonding in Organic Synthesis (Ed.: P. M. Pihko), Wiley-VCH Verlag GmbH \& Co. KGaA, Heidelberg, 2009, pp. 141-351. (b) S. J. Connon, Synlett 2009, 354-376.

[10] (a) W.-Y. Siau, J. Wang, Catal. Sci. Tech. 2011, 1, 1298-1310. (b) Y. Xi, X. Shi, Chem. Commun. 2013, 49, 8583-8585. (c) S. J. Connon, Chem. Commun. 2008, 2499-2510. (d) F. E. Held, S. B. Tsogoeva, Catal. Sci. Tech. 2016, 6, 645-667.

[11] Z. Zhang, P. R. Schreiner, Chem. Soc. Rev. 2009, 38, 1187-1198.

[12] I. T. Raheem, P. S. Thiara, E. A. Peterson, E. N. Jacobsen, J. Am. Chem. Soc. 2007, 129, 13404-13405.

[13] S. J. Connon, Chem. Eur. J. 2006, 12, 5419-5427.

[14] K. Hof, M. Lippert, P. R. Schreiner, in Science of Synthesis, Asymmetric Organocatalysis, Vol. 2, Georg Thieme Verlag, 2012, pp. 297-412.

[15] (a) C. Pérez-Casas, A. K. Yatsimirsky, J. Org. Chem. 2008, 73, 2275-2284. (b) M. Boiocchi, L. Del Boca, D. Esteban-Gomez, L. Fabbrizzi, M. Licchelli, E. Monzani, Chem. Eur. J. 2005, 11, 3097-3104.

[16] F. Aydin, N. Tunoglu, D. Aykac, Asian J. Chem. 2013, 25, 2455-2458.

[17] L. Prieto, V. Juste-Navarro, U. Uria, I. Delso, E. Reyes, T. Tejero, L. Carrillo, P. Merino, J. L. Vicario, Chem. Eur. J. 2017, 23, 2764-2768.

[18] Y.-R. Chen, G. Zhan, W. Du, Y.-C. Chen, Adv. Synth. Catal. 2016, 358, 3759-3764.

[19] It has been reported that nitrones can be activated with thioureas towards nuceophilic additions. See: T. Okino, Y. Hoashi, Y. Takemoto, Tetrahedron Lett. 2003, 44, 2817-2821

[20] P. Merino, T. Tejero, A. Diez Martinez, J. Org. Chem. 2014, 79, 2189-2202. 
[21] The concerted pathway involve a typical normal demand dipolar cycloaddition in which the dipole acts as a nucleophile. Accordingly, electron-donating groups at the nitrone-carbon should favor the reaction, which is just the opposite to that observed in Hammett plots. However, the second step of the stepwise mechanism involves a nucleophilic attack to the nitrone, which is clearly favored by the presence of electron-withdrawing groups at the nitrone carbon.

[22] The same lack of reactivity was observed with the trifluoroacetate evidencing that it is not a matter of counteranion

[23] Treatment of $7 \mathbf{a}$ with $\mathrm{Et}_{3} \mathrm{~N}$ and with a solution of a 1:1 mixture of thiourea 3 and $\mathrm{Bu}_{4} \mathrm{NOH}$ in the presence of deuterium oxide showed the same proton-deuterium exchange demonstrating that both situations render $7 \mathrm{a}$ nucleophilic, and evidencing that additional favorable circumstances should be present when the ion pair $3 \cdot 6$ is present.

[24] (a) P. Merino, T. Tejero, Synlett 2011, 1965-1977. (b) P. Merino, in Science of Synthesis. C-1 Building Blocks in Organic Synthesis (Ed.: P. W. N. M. v. Leeuwen), Georg Thieme Verlag, Stuttgart, 2014, pp. 311-331.
[25] Catalyst $\mathbf{1}$ is known to operate according to a steric model, particularly when it catalyzes Michael-type reactions. Consequently, only the attack through the less hindered $R e$ face of the iminium needs to be considered. For details see SI.

[26] The iminium ion is, actually, in equilibrium with the substrates (aldehyde and catalyst) and the intermediate hemiaminal so, it is necessary to consider the thermodynamic (and not the kinetic) of the process. Admittedly, it is necessary to establish equilibrium conditions providing enough concentration of iminium to feed the reaction. 


\section{COMMUNICATION}

A Case Study of Thiourea-Assisted Iminium Formation by Hydroxyl Anion Binding: Kinetic, Spectroscopic and Computational Evidences

Adv. Synth. Catal. Year, Volume, Page - Page

V. Juste-Navarro, L. Prieto, I. Delso, R. Manzano,

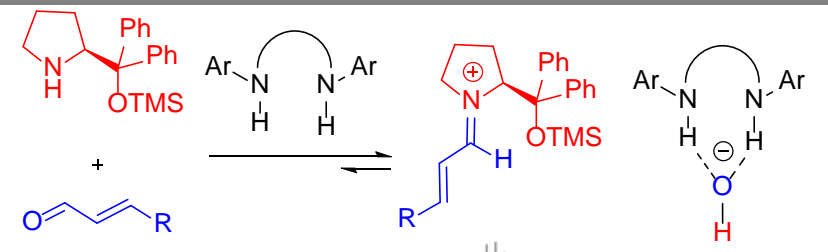

T. Tejero, E. Reyes, J. L. Vicario,* and P. Merino*

No acid co-catalyst is required

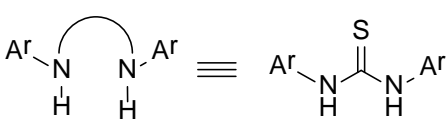<smiles></smiles> 